\title{
ÍNDICE DE QUALIDADE DE ÁGUA EM MICROBACIA SOB USO AGRÍCOLA E URBANO
}

\author{
Luís Gonzaga de Toledo*; Gilberto Nicolella \\ Embrapa Meio Ambiente, C.P. 69 - CEP: 13820-000 - Jaguariúna, SP. \\ *Autor correspondente <toledo@cnpma.embrapa.br>
}

\begin{abstract}
RESUMO: A avaliação da qualidade da água em microbacias sob diferentes usos pode ser feita pelo uso de técnicas estatísticas multivariadas. Em Guaíra (SP), foi realizado o monitoramento da qualidade da água em uma microbacia de uso agrícola e urbano com 9600 ha, no período de jun/95 a jun/96. Coletaram-se amostras de água a cada 21 dias em 5 pontos, sendo determinadas 10 variáveis (fósforo total e dissolvido, pH, oxigênio dissolvido, amônia, nitrato, condutividade elétrica, turbidez, sólidos totais em suspensão e clorofila). Calculouse um índice de qualidade de água (IQA) através do uso da técnica de análise fatorial e do método de Bartlett. Os resultados obtidos indicaram uma diferença entre os valores de IQA para as três estações a montante da cidade de Guaira e as duas estações a jusante, com valores médios de -1,757 e 2,35, respectivamente. Pela análise fatorial, oxigênio dissolvido, fósforo total, amônia e condutividade elétrica foram as variáveis que mais contribuíram na determinação do IQA. Os três primeiros fatores explicaram $71 \%$ da variância dos dados. O primeiro fator explicou $47 \%$ da variância dos dados e foi utilizado na construção do IQA. A influência climática foi pouco significativa no IQA, apresentando uma pequena deterioração na qualidade da água durante o período chuvoso. Discute-se a aplicação da técnica de análise fatorial como forma de avaliar as alterações na qualidade da água e na seleção de melhores indicadores de impacto ambiental em microbacias.
\end{abstract}

Palavras-chave: IQA, análise fatorial, monitoramento, poluição, recursos hídricos, rios

\section{WATER QUALITY INDEX FOR AGRICULTURAL AND URBAN WATERSHED USE}

\begin{abstract}
An assessment of water quality in a watershed of 9600 ha in Guaíra, SP - Brazil, submitted to different occupations (rural and urban), was carried out during the period Jun/95 to Jun/96, using multivariate statistical techniques. Water samples were collected every 21 days at five sampling sites to evaluate ten water quality parameters (total and dissolved $\mathrm{P}, \mathrm{pH}$, dissolved $\mathrm{O}_{2}, \mathrm{NH}_{4}$, nitrate, electrical conductivity, turbidity, total suspended solids, and chlorophyll). A water quality index (WQI) was determined by the factorial analysis and the Bartlett method. The results point out a significant difference of WQI between the three upstream and the two downstream (in relation to Guaíra) sampling sites (mean values of - 1.76 and 2.35 respectively). The factorial analysis indicated that dissolved $\mathrm{O}_{2}$, total $\mathrm{P}, \mathrm{NH}_{4}$, and electric conductivity were the most important parameters in determining the value of the WQI. The first three factors explained $71 \%$ of the variance of the data, whereas the first factor alone explained $47 \%$. The influence of climate parameters was of little significance for the WQI, causing a minor water quality deterioration during the rainy season. The paper discusses the applicability of the factorial analysis as a means of assessing water quality alterations, as well as for the selection of appropriate indicators for the assessment of environmental impact in watersheds.
\end{abstract}

Key words: WQI, factorial analyses, monitoring, pollution, water resources, rivers

\section{INTRODUÇÃO}

Os rios são sistemas complexos caracterizados como escoadouros naturais das áreas de drenagens adjacentes, que em princípio formam as bacias hídricas. A complexidade destes sistemas lóticos deve-se ao uso da terra, geologia, tamanho e formas das bacias de drenagem, além das condições climáticas locais.

$\mathrm{O}$ uso de indicadores de qualidade de água consiste no emprego de variáveis que se correlacionam com as alterações ocorridas na microbacia, sejam estas de origens antrópicas ou naturais.

Cada sistema lótico possui características próprias, o que torna difícil estabelecer uma única variável como um indicador padrão para qualquer sistema hídrico. Neste sentido, a busca em trabalhos de campo é a obtenção de índices de qualidade de água que reflitam resumidamente e objetivamente as alterações, com ênfase para as intervenções humanas, como o uso agrícola, urbano e industrial (Couillard \& Lefebvre, 1985).

As interações entre as diversas variáveis mensuradas numa amostra de água constitui no ponto de partida para avaliação da qualidade da água, desde que estas interações sejam obtidas de uma distribuição amostral no espaço e no tempo das variáveis do sistema a ser estudado (Harmancioglu et al., 1998).

Para uma interpretação ecológica da qualidade das águas superficiais e/ou para estabelecer um sistema de monitoramento, é necessário a utilização de métodos 
simples e que dêem informações objetivas e interpretáveis, partindo para critérios próprios que considerem as características peculiares dos recursos hídricos (Pineda \& Schäfer, 1987). Neste aspecto, o uso de índices de qualidade de água é uma tentativa que todo programa de monitoramento de águas superficiais prevê como forma de acompanhar, através de informações resumidas, a possível deterioração dos recursos hídricos ao longo da bacia hidrográfica ou ao longo do tempo.

As fontes difusas de poluição, especialmente a agricultura, têm sido objeto de atenção em muitos países devido à dificuldade de se estabelecer procedimentos de avaliação de impactos ambientais e de adotar padrões aceitáveis, como outrora ocorreu com as fontes pontuais (Sims et al., 1998; Parry, 1998). Embora estes autores enfatizem a complexidade das fontes difusas no mecanismo de transporte de fósforo em microbacias agrícolas, uma abordagem mais ampla, que envolva outras variáveis de qualidade de água deve ser considerada.

Varias técnicas para elaboração de índice de qualidade de água têm sido usadas, sendo a mais empregada aquela desenvolvida pela National Sanitation Foundation Institution e usada em países como EUA, Brasil, Inglaterra (Oliveira, 1993; Orea, 1998). Outros índices foram desenvolvidos baseados em características físico-químicas da água, como o de Liebmann, Harkins; além de índices baseados em características biológicas, comumentes associados ao estado trófico do rios.

Todos estes índices contemplam um grau de subjetividade, pois dependem da escolha das variáveis que constituirão os indicadores principais das alterações da qualidade de água. Índices baseados em técnicas estatísticas favorecem a determinação dos indicadores mais característicos do corpo de água em estudo, embora não permitam generalizações para todos os corpos de água, já que cada sistema hídrico, em princípio, possui sua característica peculiar (Haase et al., 1989). Por outro lado, como instrumento de avaliação ao longo do tempo ou do espaço, estes índices permitem acompanhar as alterações ocorridas no eixo hidrográfico.

Um dos métodos usados na formulação de índices de qualidade de água baseia-se na técnica multivariada da análise fatorial (Shoji et al., 1966; Lohani \& Mustapha, 1982; Haase \& Possoli, 1993), escolhido neste trabalho pela possibilidade de interpretação dos dados. Em resumo, tal técnica representa uma forma exploratória de conhecer o comportamento dos dados a partir de uma dimensão reduzida do espaço original dos parâmetros. Esta técnica permite selecionar as variáveis mais representativas do corpo hídrico, favorecendo a definição de indicadores mais sensíveis, tanto para adoção de um programa de monitoramento como para avaliação das alterações ocorridas nos recursos hídricos.
O presente trabalho tem por objetivo estabelecer um índice de qualidade de água resultante da composição das diversas variáveis, de acordo com a importância individual delas, de maneira a expressar de uma forma objetiva e integrada, as alterações da qualidade da água em microbacias sob diferentes usos.

\section{MATERIAL E MÉTODOS}

O trabalho de campo foi conduzido em uma microbacia de 9600 ha, localizada no município de Guaíra (SP), com uso predominantemente agrícola na porção superior e influência urbana na parte inferior. Foram monitorados cinco pontos no ribeirão Jardim, entre 20 de junho de1995 a 27 de maio de 1996. As amostras de água foram coletadas em dezoito datas, com intervalo de vinte dias entre as amostragens. $\mathrm{Na}$ Figura 1 é mostrada a distribuição das estações de coleta de água. As variáveis medidas foram fósforo total (PTOT), fósforo dissolvido $\left(\mathrm{PO}_{4}\right)$, amônia $\left(\mathrm{NH}_{4}\right)$, nitrato $\left(\mathrm{NO}_{3}\right.$ ) segundo American Public Health Association (1992). Turbidez (TURB) foi analisada em campo com turbidímetro ELLE. Sólidos totais em suspensão (STS) foi determinado por gravimetria utilizando filtro de fibra de vidro Whatmann $\mathrm{G} / \mathrm{FC}$ com $0,45 \mu \mathrm{m}$. Oxigênio dissolvido (OD), $\mathrm{pH}$, condutividade elétrica (COND) foram determinados em campo com equipamento portátil. A clorofila (CHLA) foi determinada com uso de N, N, Dimetilformamida, segundo método Moran (1982).

Utilizou-se a técnica estatística de análise fatorial na definição deste índice de qualidade de água (SAS, 1985). O modelo estatístico subjacente à análise fatorial é expresso por:

$$
z_{i j}=\sum_{p=1}^{m} a_{j p} F_{p i}+u_{j} Y_{j i} \quad(i=1,2, \ldots, N ; j=1,2, \ldots, n)
$$

em que: $a_{i p} F_{p i}=$ contribuição do fator comum $p$ à combinação linear; $u_{i} Y_{j i}=$ erro residual na representação da medida observada $z_{i j}$

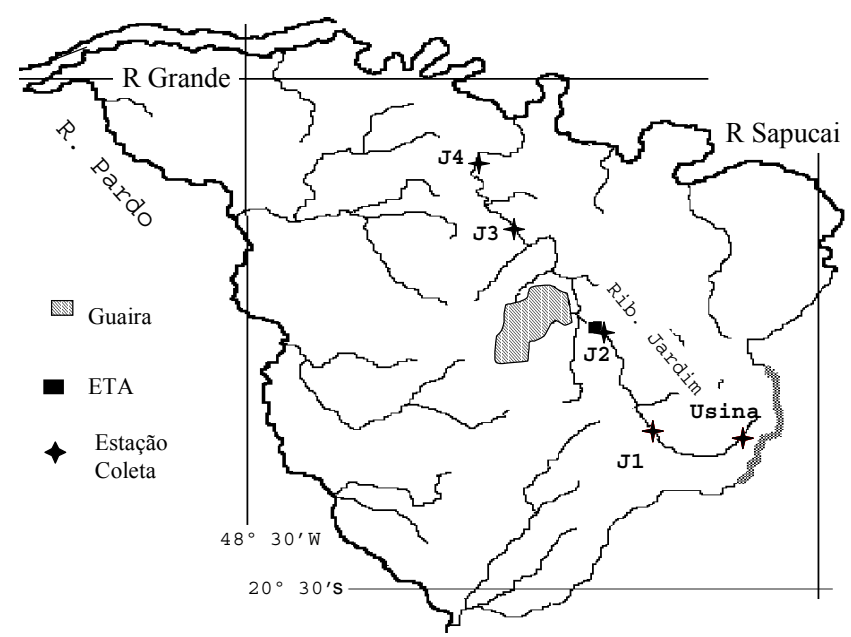

Figura 1 - Distribuição das estações de coleta de água na microbacia do Ribeirão Jardim. 
Sem qualquer perda de generalidade, pode ser assumido que os F's e os Y's têm média zero e variância unitária, visto que na prática eles são desconhecidos. Além disso, os $\mathrm{n}$ fatores únicos são supostos ser independentes e independentes dos $\mathrm{m}$ fatores comuns. No modelo anteriormente descrito, os F's são variáveis estatísticas ou variáveis aleatórias, definidos por uma função de densidade de probabilidade, que para certos propósitos é tomada como sendo normal.

A análise fatorial demanda três etapas: a) Preparação da matriz de correlação; b) Extração dos fatores comuns e a possível redução do espaço e c) rotação dos eixos relativos aos fatores comuns, visando uma solução mais simples e facilmente interpretável.

\section{RESULTADOS E DISCUSSÃO}

A variável $\mathrm{NO}_{3}$ apresentou maior média, desvio padrão e coeficiente de variação enquanto o pH mostrou menor variabilidade (Tabela 1). Estes valores representam a totalidade das estações amostradas no ribeirão Jardim, incluindo as de montante com as de jusante. Os coeficientes de variação relativamente altos são indicação da inconstância dos valores dos parâmetros ao longo do tempo e ao longo do eixo montante-jusante, influenciando de maneira relevante o índice de qualidade de água. Evidentemente, em conseqüência das intervenções antrópicas na área, essa grande variabilidade é esperada.

A extração da matriz de correlação possibilitou a escolha das variáveis mais significativas, as quais têm um papel destacado na interpretação da proposta de estudo. A dependência das variáveis medidas pode ser vista por meio da matriz de correlação (Tabela 2).

As variáveis PTOT, OD, $\mathrm{NH}_{4}$ e COND apresentaram maiores coeficientes de correlações com maior número de variáveis, ao passo que clorofila, $\mathrm{pH}$ e $\mathrm{PO}_{4}$ mostraram comportamento diametralmente oposto e, portanto, terão menor importância na composição dos fatores comuns. Entre os quatro maiores coeficientes de correlação, duas correlações negativas foram encontradas entre a variável OD-PTOT e OD-COND. Os dois maiores coeficientes positivos referem-se às variáveis $\mathrm{NH}_{4}-\mathrm{PTOT}$ e às variáveis TURB-STS. Essas constatações servem de referência quando da análise das cargas fatoriais dos fatores comuns.

A segunda etapa da análise fatorial concerne à decomposição da matriz de correlação, que baseada nas inter-relações existentes entre os dados originais, pode reduzir a dimensão do espaço das variáveis observadas, num espaço de dimensão inferior, representado pelos

TABELA 1 - Estatística descritiva básica das variáveis estudadas nos cinco pontos amostrais durante o período de jun/95 a maio/96.

\begin{tabular}{|c|c|c|c|c|c|}
\hline Parâmetro & Média & Desvio padrão & Mínimo & Máximo & Coef. Variação \\
\hline & & & & & --_ \\
\hline РTOT $\left(\mu \mathrm{gP}-\mathrm{PO}_{4} \mathrm{l}^{-1}\right)$ & 43,7 & 33,9 & 0,0 & 118,9 & 77,7 \\
\hline $\mathrm{NH}_{4}\left(\mu \mathrm{gN}-\mathrm{NH}_{4} \mathrm{I}^{-1}\right)$ & 89,8 & 125,4 & 0,0 & 455,3 & 139,6 \\
\hline $\mathrm{NO}_{3}\left(\mu \mathrm{gN}-\mathrm{NO}_{3} \mathrm{I}^{-1}\right)$ & 114,3 & 234,7 & 0,0 & 1739,2 & 205,3 \\
\hline TURB (NTU) & 11,6 & 6,2 & 2,0 & 28,3 & 53,3 \\
\hline STS (mg L-1) & 11,2 & 6,9 & 0,0 & 37,0 & 61,2 \\
\hline $\mathrm{PO}_{4}\left(\mu \mathrm{gP}-\mathrm{PO}_{4} \mathrm{I}^{-1}\right)$ & 7,8 & 6,8 & 0,0 & 40,6 & 86,4 \\
\hline $\mathrm{OD}\left(\mathrm{mg} \mathrm{L}^{-1}\right)$ & 4,6 & 2,1 & 0,0 & 8,1 & 54,8 \\
\hline $\mathrm{pH}$ & 6,7 & 0,4 & 5,5 & 7,1 & 6,7 \\
\hline $\operatorname{COND}\left(\mu \mathrm{S} \mathrm{cm}^{-1}\right)$ & 43,8 & 10,8 & 27,0 & 68,9 & 24,5 \\
\hline CHLA ( $\mu$ gChla L L $\left.{ }^{-1}\right)$ & 7,4 & 4,6 & 0,9 & 26,7 & 62,3 \\
\hline
\end{tabular}

Tabela 2 - Matriz de correlação das variáveis de qualidade de água no Ribeirão Jardim.

\begin{tabular}{|c|c|c|c|c|c|c|c|c|c|c|}
\hline Variável & PTOT & $\mathrm{NH} 4$ & NO3 & TURB & STS & PO4 & OD & $\mathrm{pH}$ & COND & CHLA \\
\hline РTOT & 1,000 & & & & & & & & & \\
\hline $\mathrm{NH}_{4}$ & 0,757 & 1,000 & & & & & & & & \\
\hline $\mathrm{NO}_{3}$ & 0,502 & 0,381 & 1,000 & & & & & & & \\
\hline TURB & 0,569 & 0,388 & 0,148 & 1,000 & & & & & & \\
\hline STS & 0,486 & 0,362 & 0,081 & 0,784 & 1,000 & & & & & \\
\hline $\mathrm{PO}_{4}$ & 0,535 & 0,539 & 0,618 & 0,139 & 0,128 & 1,000 & & & & \\
\hline OD & $-0,677$ & $-0,620$ & $-0,100$ & $-0,399$ & $-0,437$ & $-0,283$ & 1,000 & & & \\
\hline $\mathrm{pH}$ & $-0,390$ & $-0,399$ & $-0,174$ & $-0,326$ & $-0,190$ & $-0,275$ & 0,509 & 1,000 & & \\
\hline COND & 0,601 & 0,671 & 0,246 & 0,433 & 0,489 & 0,323 & $-0,709$ & $-0,276$ & 1,000 & \\
\hline CHLA & 0,299 & 0,379 & 0,265 & 0,370 & 0,319 & 0,151 & $-0,057$ & $-0,198$ & 0,169 & 1,000 \\
\hline
\end{tabular}


fatores comuns. Foram obtidos, pelo método das componentes principais, três fatores maiores do que a unidade associados às raízes características, os quais explicam $71,33 \%$ da variância total das variáveis originais.

Os resultados das estimativas das cargas fatoriais e das comunalidades encontram-se na Tabela 3. O primeiro fator explica $44,49 \%$ da variabilidade dos dados. Este fator pode ser associado a qualidade da água, desde que todas as variáveis com cargas positivas estão corroborando para a baixa qualidade da água, o oposto ocorrendo com as variáveis com cargas negativas, particularmente e de modo expressivo a variável $\mathrm{OD}$.

Da observação das comunalidades, vê-se que $88,4 \%$ da variância da variável OD é explicada pelos três fatores comuns, enquanto apenas $29,3 \%$ da variância do $\mathrm{pH}$ é explicada por esses mesmos fatores. O complemento desses valores, denominado unicidade, indica o quanto os fatores comuns falham em explicar a variância total unitária da variável.

O fator 1 representa a parte comum mais abrangente das variáveis observadas, podendo ser associado ou nomeado como índice de qualidade de água (IQA). Adotou-se o procedimento "no rotate", já que outros procedimentos de rotação dos eixos (varimax, equimax e quartimax) não produziram resultados mais adequados para estarem associados a um índice de qualidade água.

Após a avaliação dos resultados da análise fatorial é possível a construção de um ou mais fatores de escala a partir da constatação da existência de determinadas dimensões subjacentes aos dados. $O$ interesse é examinar os dados amostrais em termos dessas dimensões, ao invés de examinarmos cada variável separadamente.

Neste trabalho foi utilizado o método de Bartlett para a criação dos fatores de escala, que minimiza a

Tabela 3 - Matriz das cargas fatoriais, variância observada e comunalidade das variáveis de qualidade de água do Ribeirão Jardim.

\begin{tabular}{lcccc}
\hline \multirow{2}{*}{ Variável } & \multicolumn{3}{c}{ Matriz de carga fatorial } & \multirow{2}{*}{$\begin{array}{c}\text { comunalidade } \\
\text { Estimada }\end{array}$} \\
\cline { 2 - 4 } & $\mathrm{F} 1$ & $\mathrm{~F} 2$ & \multicolumn{1}{c}{$\mathrm{F} 3$} & 0,732 \\
\hline PTOT & 0,856 & 0,009 & 0,029 & 0,758 \\
$\mathrm{NH}_{4}$ & 0,839 & 0,205 & $-0,111$ & 0,795 \\
$\mathrm{NO}_{3}$ & 0,464 & 0,693 & 0,304 & 0,802 \\
TURB $_{\text {STS }}^{0,705}$ & $-0,461$ & 0,305 & 0,797 \\
$\mathrm{PO}_{4}$ & 0,666 & $-0,525$ & 0,278 & 0,797 \\
OD & 0,547 & 0,670 & $-0,042$ & 0,751 \\
pH & $-0,781$ & 0,153 & 0,500 & 0,884 \\
COND & $-0,404$ & 0,048 & 0,357 & 0,293 \\
CHLA & 0,776 & $-0,081$ & $-0,266$ & 0,678 \\
\hline Variância(\%) & 44,49 & 59,49 & 71,33 & 0,643 \\
\hline
\end{tabular}

Scientia Agricola, v.59, n.1, p.181-186, jan./mar. 2002 variância do erro, sendo os escores preditos pelo modelo:

$$
\hat{\mathrm{F}}=\mathrm{XU}^{-2} B\left(B U^{-2} B\right)^{-1}
$$

onde: $X=$ vetor das variáveis observadas; $U^{-2}=$ matriz diagonal das variâncias únicas; $B=$ matriz das cargas fatoriais.

No caso particular do critério de Bartlett, os coeficientes estimados que comporão o índice de qualidade de água, são expressos pela equação:

$I Q A=\hat{\mathrm{F}}=0.152 Z_{1}+0.166 Z_{2}+0.181 Z_{3}+0.169 Z_{4}+$ $0.157 Z_{5}-0.077 Z_{6}-0.322 Z_{7}+0.031 Z_{8}+0.081 Z_{9}+$ $0.057 Z_{10}$

Nessa expressão, os $Z_{i}$ são variáveis padronizadas e os sub-índices representam as variáveis na mesma ordem (Tabela 3). Os primeiros cinco coeficientes têm influência mais acentuada na composição do valor do índice que os três últimos e estão associados à variáveis que aumentam com a poluição, ao passo que a variável $Z_{7}$ é a que tem mais influência na obtenção de um valor de índice negativo. O IQA calculado por este procedimento possui média zero e variância unitária, estando compreendido entre -3 e 3 , denotando os valores negativos como os de melhor qualidade.

Os valores calculados de IQA, para cada ponto de amostragem, num total de cinco, ao longo do tempo, denominados escores fatoriais, são apresentados na Figura 2.

Rodrigues (1998), utilizando de índices de qualidade de água em córregos do município de Guaíra, obteve uma diferenciação entre as estações de montante e jusante, sem entretanto avaliar as alterações da qualidade da água ao longo do tempo. Isto ocorre porque o autor utilizou índice baseado em escores de agregação. Das variáveis utilizadas por este autor, o oxigênio dissolvido foi determinante na

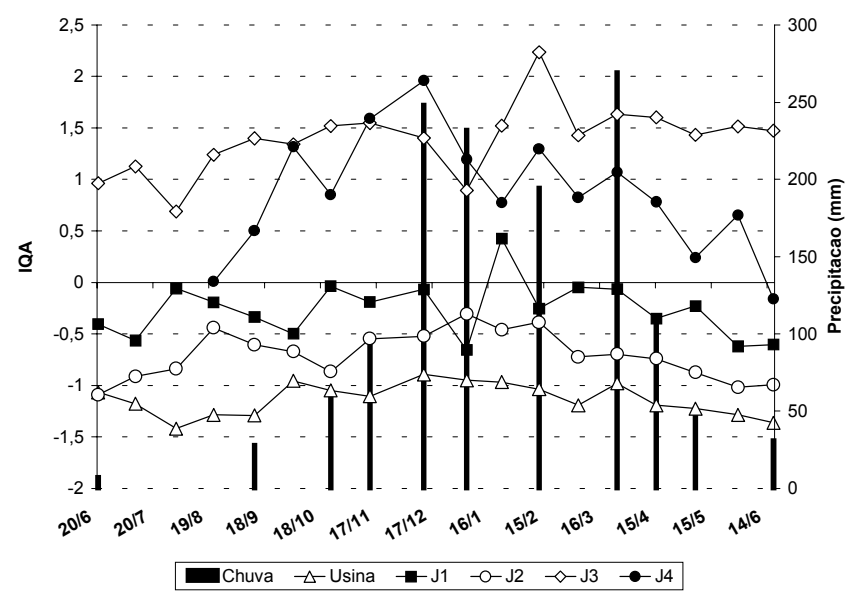

Figura 2 - Distribuição do valores de IQA nos pontos de amostragens do Ribeirão Jardim no período de junho de 95 a junho de 96. 
definição do IQA. Isto corrobora os resultados apresentados, onde o OD também teve o terceiro maior escore fatorial em termos absolutos $(-0,781)$, influenciando negativamente o IQA.

O aporte de material no ribeirão Jardim ocorre devido as dois motivos principais: efluentes urbanos da cidade de Guaíra, que alteram o IQA nas estações J3 e $\mathrm{J} 4$ e o escorrimento originado das áreas agrícolas, os quais influenciam as estações J1 e J2 (Figura 3). A entrada de material originado das áreas agrícolas e de fontes urbanas contribui para o aumento na carga de fósforo e amônia e promove decréscimo na concentração de OD, devido ao consumo deste elemento nos processos respiratórios da comunidade hídrica, principalmente se for material orgânico. Associado a este processo de mineralização de material orgânico, houve aumento na condutividade elétrica da água, que pode ser observado pela relação negativa entre este parâmetro e a variável $\mathrm{OD}$.

Como o IQA representa uma variável estatística, com contribuições preponderantes das variáveis primárias PTOT, $\mathrm{NH}_{4}$, OD e COND, as variações do IQA mostrou-se bastante associadas às alterações da qualidade da água, ao contrário de estudarmos isoladamente cada variável.

Diferentes usos da terra ao longo do eixo hídrico provocam alterações nas relações entre as variáveis. No trecho de montante, onde o uso agrícola predomina, a influência das variáveis TURB e STS foi mais significativa devido à ocorrência de processos erosivos, mascarando as relações entre PTOT, OD e $\mathrm{NH}_{4}$, dominantes nos trecho a jusante. Ferreira et al. (1996) mostraram evidências indiretas da influência dos sólidos em suspensão na estação $\mathrm{J} 2$, indicando uma relação positiva entre STS e o consumo de sulfato de alumínio pela estação de tratamento de água do município de Guaíra. Estes autores sugerem que os sedimentos em suspensão originam-se a partir das áreas agrícolas devido aos processos erosivos.

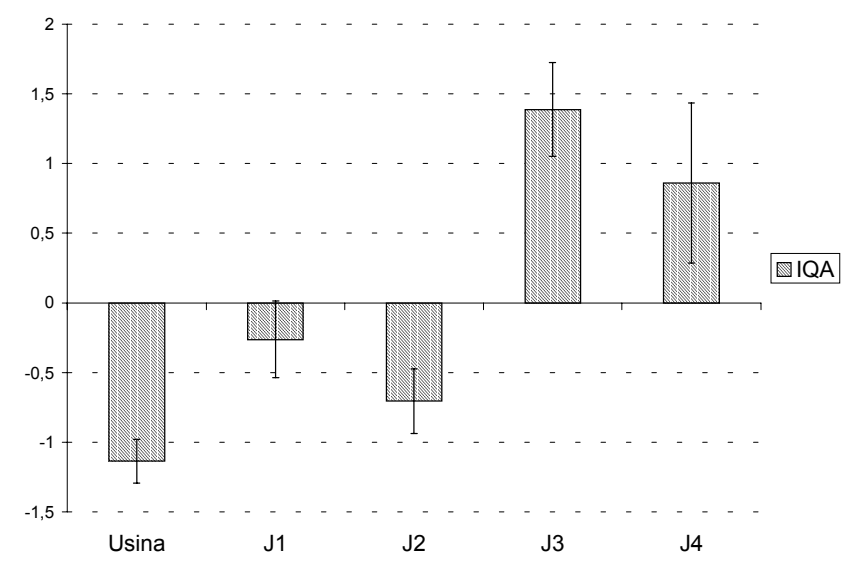

Figura 3 - Valores médios e desvios padrões do IQA nos pontos de amostragens do Ribeirão Jardim.
O papel do fósforo na eutrofização dos recursos hídricos é essencial, e a origem deste nutriente a partir de áreas agrícolas tem sido colocada em relevância como indicador de qualidade de água, já que outros indicadores como sólidos em suspensão e turbidez estão associados ao transporte de fósforo (Parry, 1998).

Sharpley \& Rekolainen (1997) e Rekolainen et al. (1997) indicam que o aporte de fósforo aos recursos hídricos tem como principal agente o uso urbano, seguido pelo uso agrícola do solo. Neste trabalho ficou evidente a influência da concentração de fósforo total na deterioração da qualidade da água, sendo o uso urbano o principal agente causador, representado pelas estações de coleta J3 e J4.

A influência do uso urbano da microbacia na região de jusante (estações $\mathrm{J} 3$ e J4) foi reforçada pela entrada do tributário Córrego da Santa Cruz, afluente do ribeirão Jardim. Este tributário recebe o efluente proveniente de uma pocilga, com produção de cerca de 500 cabeças/ano, sem tratamento dos dejetos e deságua no ribeirão Jardim antes da estação J3. O enriquecimento em nutrientes e sais reforçaram as relações entre as variáveis OD-PTOT- $\mathrm{NH}_{4}$, produzindo um IQA médio mais alto na estação $\mathrm{J} 3$, o que corrobora a deterioração na qualidade da água. Nota-se também melhoria na qualidade da água na estação $\mathrm{J} 4$ devido ao processo de auto depuração ocorrido no trecho entre J3 e J4.

A ocorrência de precipitação sobre a qualidade da água não foi significativa (Figura 2). Entretanto, uma pequena deterioração na qualidade de água foi observada nos meses de chuva, devido ao aumento do IQA, principalmente nas estações de montante (Usina e J1). Considerando o tamanho da bacia hidrográfica estudada, a ocorrência de chuvas associadas às amostragens de água podem não terem ocorridas concomitantemente, o que pode ter prejudicado as relações entre as variáveis mais relacionadas ao escoamento superficial, as quais seriam, turbidez e STS. Deve-se ter em mente que os valores de precipitação constituem o total no mês. O intervalo entre os eventos climáticos e as alterações na qualidade de água é diretamente proporcional ao tamanho das microbacias, sendo que em rios de pequeno porte este intervalo pode situar-se entre 12 a 36 horas (Meybeck et al., 1996). Segundo Lennox et al. (1997) as coletas mensais ou quinzenais em rios de pequeno porte tendem a representar, em $80 \%$ das vezes, as características de qualidade de água do fluxo de base.

Outra consideração diz respeito a representavidade espacial das medições pluviométricas em estudos de pequenas áreas. Em maio de 96, a ocorrência de chuvas torrenciais localizadas em uma pequena porção do município de Guaíra afetou marcadamente a qualidade da água de um outro afluente, não sendo este fato observado no Ribeirão Jardim. 


\section{CONCLUSÕES}

Os valores de IQA para Ribeirão Jardim, calculados pela técnica de análise fatorial, mostraram diferenças significativas entre pontos de coleta de água a montante e a jusante do município de Guaíra.

Os principais indicadores de qualidade de água que influenciaram a composição do IQA foram as variáveis $\mathrm{OD}, \mathrm{PTOT}, \mathrm{NH}_{4}$ e COND.

O uso da técnica de análise fatorial permitiu também avaliar as condições de deterioração da qualidade de água para a região de Guaíra, com vistas à obtenção de indicadores específicos, os quais poderão ser monitorados ao longo do tempo.

\section{REFERÊNCIAS BIBLIOGRÁFICAS}

AMERICAN PUBLIC HEALTH ASSOCIATION. Standard methods for the examination of water and wastewater. 18.ed. Baltimore: APHA, 1992. 1v.

COUILLARD, D.; LEFEBVRE, Y. Analysis of water quality indices. Journal of Environmental Management, v.21, p.161-179, 1985.

FERREIRA, C.J.A.; LUCHIARI JR., A.; TOLEDO, L.G.; LUIZ, A.J.B.; ROCHA, J.; LELIS, L.L. Influência dos sistemas agrícolas irrigados por aspersão sobre a qualidade dos recursos hídricos. In: CONGRESSO NACIONAL DE IRRIGAÇÃO E DRENAGEM, 11., Campinas, 1996. Anais. Campinas: ABID, 1996. p.467-479.

HAASE, J.; POSSOLI, S. Estudo da utilização da técnica de análise fatorial na elaboração de um índice de qualidade de água: comparação entre dois regimes hidrológicos diferentes, RS. Acta Limnologica Brasiliensia, v.6, p.245-255, 1993.

HAASE, J.; KRIEGER, J.A.; POSSOLI, S. Estudo da viabilidade do uso da técnica fatorial como um instrumento na interpretação de qualidade das águas da bacia hidrográfica do Guaiba, RS, Brasil. Ciência e Cultura, v.41, p.576-582, 1989.

HARMANCIOGLU, N.B.; OZKUL, S.A.; ALPASLAN, M.N. Water monitoring and network design. In: HARMANCIOGLU, N.B.; SINGH, V.P.; ALPASLAN, M.N. (Ed.) Environmental data management. The Hague: Kluwer Academic Publishers, 1998. p.61-100. (Water Science Technology Library, 27).

LENNOX, S.D.; FOY, R.H.; SMITH, R.V.; JORDAN, C. Estimating the contribution from agriculture to the phosphorus load in surface water. In: TUNNEY, H.; CARTON, O.T.; BROOKES, P.C.; JOHNSTON, A.E. (Ed.) Phosphorus loss from soil to water. New York: CAB International, 1997. p.55-75.
LOHANI, B.N.; MUSTAPHA, N. Indices for water quality assessment in river: a case study of the Linggi river in Malaysia. Water Supply and Managment, v.6, p.545-555, 1982.

MEYBECK, M.; FRIEDRICH, G.; THOMAS, R.; CHAPMAN, D. River. In: CHAPMAN, D. (Ed.) Water quality assessments. London: UNESCO; WHO; UNEP, 1996. p.241-320.

MORAN, R. Formulae for determination of chlorophyllous pigments extracted with N,N-dimethylformamide. Plant Physiology, v.69, p.1376-1381, 1982.

OLIVEIRA, S. (Coord.) Relatório de qualidade ambiental no Estado de São Paulo - 1993. São Paulo: CETESB, 1994. 50p. (Série Relatórios).

OREA, D.G. Evaluación de impacto ambiental. Madrid: Editorial Agrícola Española, 1998. 260p.

PARRY, R. Agriculture phosphorus and water quality: a U.S. Environmental Protection Agency perspective. Journal of Environmental Quality, v.27, p.258-261, 1998.

PINEDA, M.D.; SCHÄFER, A. Adequação de critérios e métodos de avaliação da qualidade de águas superficiais baseada no estudo ecológico do rio Gravatai, Rio Grande do Sul, Brasil. Ciência e Cultura, v.39, p.198-206, 1987.

REKOLAINEN, S.; KHOLM, P.E.; ULEN, B.; GUSTAFSON, A. Phosphorus losses from agriculture to the surface water in the nordic countries In: TUNNEY, H.; CARTON, O.T.; BROOKES, P.C.; JOHNSTON, A.E. (Ed.) Phosphorus loss from soil to water. New York: CAB International, 1997. p.7793.

RODRIGUES, G.S. Avaliação de impactos ambientais em projetos de pesquisas - Fundamentos, princípios e introdução à metodologia. Jaguariúna: EMBRAPA, CNPMA, 1998. 66p. (Documentos, 14).

SAS INSTITUTE. SAS user's guide: statistics. version 5. Cary, 1985. 1v.

SHARPLEY, A.N.; REKOLAINEN, S. Phosphorus in agriculture and its environmental implications. In: TUNNEY, H.; CARTON, O.T.; BROOKES, P.C.; JOHNSTON, A.E. (Ed.) Phosphorus loss from soil to water. New York: CAB International, 1997. p.1-53.

SHOJI, H.; YAMANOTO, T.; NAKAMURA, T. Factor analysis on stream pollution of the Yodo River systems. Air and Water Pollution, v.10, p.291-299, 1966.

SIMS, J.T.; SIMARD, R.R.; JOERN, B.C. Phosphorus loss in agricultural drainage: historical perspective and current research. Journal of Environmental Quality, v.27, p.277293, 1998.

$\overline{\text { Recebido em } 30.10 .00}$ 